\title{
Pneumomediastinum and Diffuse Subcutaneous Emphysema with SARS-CoV-2 Infection Requiring Operative Management for Impending Airway Collapse: A Case Report
}

Kevin Lin ${ }^{1}$, Chris Stefaniak ${ }^{2}$, Connor Bunch ${ }^{1}$, Robert March ${ }^{2}$, Mahmud Zamlut ${ }^{2}$, Syed Raza $^{2}$, Walter Osario ${ }^{2}$, Josh Korzan ${ }^{2}$, Jeffery Show ${ }^{2}$, Nicolas Mjaess ${ }^{2}$, Shivani Patel ${ }^{2}$, Sufyan Zackariya ${ }^{2}$, Ali Sualeh ${ }^{2}$, Grant Wiarda ${ }^{2}$, Hamid Al-Fadhl ${ }^{2}$, Anthony Thomas ${ }^{1}$, Rashid Khan ${ }^{3}$, Laura Gillespie ${ }^{2}$, and Mark Walsh ${ }^{1}$

${ }^{1}$ Indiana University School of Medicine-South Bend

${ }^{2}$ Saint Joseph Regional Medical Center Mishawaka Campus

${ }^{3}$ Michiana Hematology Oncology PC

March 30, 2021

\begin{abstract}
Pneumomediastinum is a rare complication of severe acute respiratory syndrome coronavirus 2 (SARS-CoV-2) infection that has increased in incidence with the novel coronavirus disease 2019 pandemic. Although traditionally managed with conservative measures, we present the indications and methods for the first operative management of tension pneumomediastinum with concomitant SARS-CoV-2 infection.
\end{abstract}

Title : Pneumomediastinum and Diffuse Subcutaneous Emphysema with SARS-CoV-2 Infection Requiring Operative Management for Impending Airway Collapse: A Case Report

Authors : Kevin P. Lin BS ${ }^{1}$, Christopher Stefaniak BS${ }^{2}$, Connor M. Bunch BS ${ }^{1}$, Robert March $\mathrm{MD}^{3}$, Mahmud Zamlut $\mathrm{MD}^{4}$, Syed Raza $\mathrm{MD}^{4}$, Walter Osorio BSN ${ }^{4}$, Josh Korzan $\mathrm{BS}^{4}$, Jeffery Show MD ${ }^{5}$, Nicolas Mjaess $^{5}$, Shivani Patel ${ }^{5}$, Sufyan Zackariya ${ }^{5}$, Ali Sualeh ${ }^{5}$, Grant Wiarda ${ }^{5}$, Hamid Al-Fadhl ${ }^{5}$, Anthony V. Thomas $\mathrm{BS}^{1}$, Rashid Z. Khan $\mathrm{MD}^{6}$, Laura Gillespie PharmD ${ }^{7}$, Mark M. Walsh* ${ }^{*} D^{1,5}$

\footnotetext{
Affiliations :

${ }^{1}$ Indiana University School of Medicine South Bend Campus, Notre Dame, Indiana, USA

${ }^{2}$ Department of Medical Affairs, St. Joseph Regional Medical Center, Mishawaka, Indiana, USA

${ }^{3}$ Department of Cardiothoracic Surgery, St. Joseph Regional Medical Center, Mishawaka, Indiana, USA

${ }^{4}$ Department of Intensive Care Medicine, St. Joseph Regional Medical Center, Mishawaka, Indiana, USA

${ }^{5}$ Departments of Emergency Medicine and Internal Medicine, St. Joseph Regional Medical Center, Mishawaka, Indiana, USA

${ }^{6}$ Department of Hematology, Michiana Hematology Oncology, Mishawaka, Indiana, USA

${ }^{7}$ Department of Quality Assurance and Performance Improvement, St. Joseph Regional Medical Center, Mishawaka, Indiana, USA
} 
*Corresponding Author : Mark M. Walsh MD, Departments of Emergency Medicine and Internal Medicine, Saint Joseph Regional Medical Center, 5215 Holy Cross Parkway, Mishawaka, Indiana 46545 (e-mail: markwalshmd@gmail.com).

Emails :

Kevin P. Lin kevlin@iu.edu

Christopher Stefaniak chris.stefaniak@sjrmc.com

Connor M. Bunch cmbunch@iu.edu

Robert March robert.March@sjrmc.com

Mahmud Zamlut mzamlut@gmail.com

Syed Raza dochussain@hotmail.com

Walter Osorio walter.osorio1990@gmail.com

Josh Korzan korzanjl@gmail.com

Jeffery Show jshow@alumni.nd.edu

Nicolas Mjaess nmjaess@nd.edu

Shivani Patel ssp@iu.edu

Sufyan Zackariya szackari@iu.edu

Ali Sualeh asualeh@iu.edu

Grant Wiarda grwiarda@gmail.com

Hamid Al-Fadhl halfadhl@iu.edu

Anthony V. Thomas 33thomasa@gmail.com

Rashid Z. Khan rkhan@mhopc.com

Laura Gillespie gillesla@sjrmc.com

Mark M. Walsh markwalshmd@gmail.com

Abstract

Pneumomediastinum is a rare complication of severe acute respiratory syndrome coronavirus 2 (SARS-CoV2) infection that has increased in incidence with the novel coronavirus disease 2019 pandemic. Although traditionally managed with conservative measures, we present the indications and methods for the first operative management of tension pneumomediastinum with concomitant SARS-CoV-2 infection.

\section{Key Clinical Message}

COVID-19 patients who develop pneumomediastinum and become acutely unstable may require surgical intervention. Complete clinical healing of impending airway collapse was achieved by anterior mediastinal and pericardial drainage.

\section{Keywords}

pneumomediastinum, COVID-19, mediastinal emphysema, drainage, surgery, pericardial window, coronavirus, case report

\section{Introduction}


Pneumomediastinum is a rare complication in patients infected with the severe acute respiratory syndrome coronavirus 2 (SARS-CoV-2). Pneumomediastinum typically resolves with conservative management. Rarely, airway collapse, persistent hemodynamic instability, or progression to cardiac tamponade may require surgical intervention. ${ }^{(1,2)}$ Described here is a unique case of SARS-CoV-2 infection requiring invasive ventilation that was later complicated by tension pneumomediastinum, which ultimately required operative anterior mediastinal and pericardial drainage. The scant literature on this phenomenon was reviewed, and we discuss the indications and methods for the first operative management of tension pneumomediastinum in a patient with the novel coronavirus disease 2019 (COVID-19). ${ }^{(1)}$

\section{Case History}

A 48-year-old male presented to the emergency department six days after onset of shortness of breath, cough, and chills. He tested positive for COVID-19 five days prior. On admission, vital signs showed blood pressure of $116 / 80 \mathrm{mmHg}$, temperature of 99.8 Fahrenheit, heart rate of 109 beats per minute, respiratory rate of 40 breaths per minute, and oxygen saturation of $72 \%$ on room air. The patient was initially placed on 6 $\mathrm{L} / \mathrm{min}$ oxygen by nasal cannula and escalated to $15 \mathrm{~L} / \mathrm{min}$. Serum labs on admission were significant for sodium of $126 \mathrm{mg} / \mathrm{dL}$ (standard range 135-145), potassium of $3.4 \mathrm{mg} / \mathrm{dL}$ (standard range 3.6-5.2), C-reactive protein of $429 \mathrm{mg} / \mathrm{L}$ (standard range 0-10.0), lactate of $2.3 \mathrm{mMol} / \mathrm{L}$ (standard range 0.4-2.0), leukocytosis of 18,860 cells/L (standard range 4,000-11,000), and D-dimer of $1.52 \mathrm{mg} / \mathrm{L}$ (standard range $0-0.53$ ). He was confirmed COVID-19 positive by reverse transcriptase-polymerase chain reaction of nasopharyngeal swab. Initial imaging revealed diffuse bilateral ground-glass infiltrates above both lung fields which was subsequently described as "tree-in-bud" acute respiratory distress syndrome. (Figure 1) Four-extremity Doppler ultrasound revealed a right upper extremity brachial vein deep vein thrombus (DVT), and the patient was placed on once daily $1 \mathrm{mg} / \mathrm{kg}$ subcutaneous enoxaparin.

On the same day as admission, the patient continued to have tachycardia, tachypnea, and low oxygen saturation despite high flow oxygen by nasal cannula and required endotracheal intubation and mechanical ventilation for the next 48 hours due to respiratory insufficiency. The patient self-extubated on day four of hospitalization and remained tachypneic until the last twelve hours of hospitalization. He was discharged on day ten and prescribed apixaban $5 \mathrm{mg}$ twice daily for upper extremity DVT therapy. The patient did not require oxygen supplementation at discharge.

Three days after discharge, the patient developed a sense of fullness in his neck. Five days after discharge, his family physician made a house call and noticed that the patient had resting tachycardia at 108 beats per minute, oxygen saturation of $93 \%$ on room air, and a heart rate that increased to 140 while walking in place for one minute. The patient had faint crackles over the right posterior lung field but no evidence of subcutaneous crepitus at this time.

On the seventh day following discharge and two days after the physician house call, the patient returned to the emergency department with increasing swelling in the neck and a sensation of "crackling" in his neck, chest, and scrotum. Physical exam revealed a blood pressure of 120/70 $\mathrm{mmHg}$, a heart rate of 112 beats per minute at rest, oxygen saturation of $94 \%$ on room air, a respiratory rate of 28 breaths per minute, and a temperature of 99.6 Fahrenheit. Physical exam revealed increased swelling of the neck compared to two days prior, palpable crepitus in the neck, chest, and abdomen, and diminished breath sounds over both lung bases. There was no audible Hamman's crunch sign of a pericardial friction rub. A computed axial tomography (CT) scan revealed diffuse subcutaneous emphysema in the neck and chest with massive pneumomediastinum and marked improvement of the parenchymal infiltrates (Figure 2).

The patient was admitted to the hospital for monitoring. Forty-eight hours later, the patient's subcutaneous emphysema spread to his arms as demonstrated by newly palpable crepitus from his axilla to the wrists. Additionally, there was significantly increased swelling and crepitus in the neck. The patient described difficulty with breathing due to a sense of local constriction in his upper airway with progressively increased stridor and increased pitch of his voice that caused great difficulty with speaking and breathing.

With concern for impending airway obstruction, the patient was taken for emergency mediastinal drainage. 
He received a subxiphoid pericardial window, subxiphoid and suprasternal drainage of the pneumomediastinum, substernal dissection with a lighted scope, and laryngotracheobronchoscopy. A suprasternal notch transverse incision and dissection to the anterior mediastinum was also performed. A lighted balloon tipped endoscope was used to further develop the substernal space from the subxiphoid space up to the suprasternal notch. Neither pleural space was entered. The anterior mediastinum was completely decompressed, and a Blake drain (24 French) was placed and exited through a separate space in the subxiphoid region (Figure 3). A pericardial wound was then created in this space and was drained as well with a Blake drain (24 French). Fiberoptic bronchoscopy was then conducted to confirm that there were no injuries extending down to the major bronchi. A repeat ultrasound of the upper extremity post-operatively demonstrated a complete disappearance of the brachial vein thrombus present only two weeks prior. The patient made an uneventful recovery with complete clinical and radiographic healing.

\section{Discussion}

Tension pneumomediastinum is a rare but potentially lethal condition seen in critically ill patients. Traditionally, pneumomediastinum occurs in young patients with asthma. During an asthmatic attack, rapid breathing causes alveolar rupture into the lower-pressure mediastinum. This condition is often harmless and resolves spontaneously as air is absorbed with time. ${ }^{(3)}$ On the other hand, tension pneumomediastinum can also occur due to prolonged mechanical ventilation, particularly in settings of high end-expiratory pressure. ${ }^{(2)}$ Understandably so, this complication has seen an increase in incidence following the emergence of the COVID-19 pandemic, as high end-expiratory pressure ventilation has been utilized to a greater extent for management of COVID-19-related respiratory distress. ${ }^{(1)}$ This form of pneumomediastinum is far more complicated and requires urgent intervention.

Tension pneumomediastinum is thought to occur in patients with COVID-19 secondary to diffuse alveolar damage. The increased presence of diseased alveoli on the mediastinal surface allows for preferential rupture into the mediastinum due to the pressure gradient between the alveoli and the perivascular sheaths. Further spreading of the pulmonary interstitial emphysema into the mediastinum is subsequently known as the Macklin effect. ${ }^{(4)}$ In patients with COVID-19, the diseased lung may create a one-way valve at the mediastinal/pleural border, which can subsequently lead to air retention in the mediastinum. Increased pressure in the mediastinum can cause compress mediastinal contents. In particular, compression of the great vessels can lead to decreased venous return, hypotension with tachycardia, and potentially cardiovascular collapse. ${ }^{(2)}$

Currently, management for tension pneumomediastinum in the COVID-19 population has largely been conservative. ${ }^{(1)}$ Different approaches include reducing airway pressures and adjusting ventilator settings to allow for permissive hypercapnia in an effort to reduce pressure gradients across the mediastinal surface. These methods may be sufficient for management of tension pneumomediastinum in stable COVID-19 patients, but those who are unstable may require immediate surgical decompression. After review of the current literature, we describe the first case report of operative management for a massive tension pneumomediastinum secondary to COVID-19.(1) Of note, there was one previous report of tension pneumomediastinum secondary to COVID-19 that resolved with bedside mediastinotomy via the Chamberlain procedure. ${ }^{(5)}$

In our patient with COVID-19, a tension pneumomediastinum formed in the chest and neck, with subsequent spread to the arms bilaterally. The enlarging pneumomediastinum caused difficulty breathing and progressive dysphonia with an increased pitch in the tone of his voice. Due to impending airway obstruction, the patient was sent for emergent mediastinal drainage. Specifically, we created a subxiphoid pericardial window, employed subxiphoid and suprasternal drainage of the pneumomediastinum, and performed substernal dissection with lighted scope. The anterior mediastinum was decompressed completely using our operative procedure, resulting in rapidly reduced swelling in the patient's neck, improvement of his voice, and disappearance of the crepitus with complete clinical and radiographic healing.

Here, we describe the first operative management of massive tension pneumomediastinum secondary to SARS-CoV-2 infection. In this case of a 48-year-old male with severe COVID-19 pneumonitis requiring intubation, we used an operative technique that provided rapid decompression of unstable tension pneumo- 
mediastinum using a pericardial window and mediastinal drain. This case demonstrates that precipitous decline may occur in a patient with diseased lung parenchyma such as COVID-19 and that our method may offer an effective operative solution for rapid decompression required for massive tension pneumomediastinum dissolution.

\section{Abbreviations}

Computed Axial Tomography (CT)

Deep Vein Thrombosis (DVT)

Novel Coronavirus Disease 2019 (COVID-19)

Severe Acute Respiratory Syndrome Coronavirus 2 (SARS-CoV-2)

\section{Declarations}

\section{Consent for Publication}

Informed consent was obtained from the patient to publish the case report.

\section{Acknowledgments}

Not applicable.

\section{Conflicts of Interest Statement}

The authors declare that they have no competing interests.

\section{Funding}

No funding was obtained for this publication.

\section{Authors' Contributions}

KPL: Conceptualization of manuscript, literature review, review of manuscript, approval of final draft.

CS: Conceptualization of manuscript, literature review, review of manuscript, approval of final draft.

CMB: Literature review, review of manuscript, approval of final draft

RM: Conceptualization of manuscript, review of manuscript, approval of final draft

MZ: Conceptualization of manuscript, review of manuscript, approval of final draft

SR: Collection of data, review of manuscript, approval of final draft

WO: Collection of data, review of manuscript, approval of final draft

JK: Collection of data, review of manuscript, approval of final draft

JS: Collection of data, review of manuscript, approval of final draft

NM: Literature review, review of manuscript, approval of final draft

SP: Literature review, review of manuscript, approval of final draft

SZ: Literature review, review of manuscript, approval of final draft

AS: Literature review, review of manuscript, approval of final draft

GW: Literature review, review of manuscript, approval of final draft

HA: Literature review, review of manuscript, approval of final draft

AVT: Review of manuscript, approval of final draft 
RK: Conceptualization of manuscript, review of manuscript, approval of final draft.

LG: Conceptualization of manuscript, review of manuscript, approval of final draft.

MMW: Conceptualization of manuscript, literature review, review of manuscript, approval of final draft.

\section{Ethics Approval and Consent to Participate}

This protocol development was approved by the Institutional Review Board of Saint Joseph Regional Medical Center at Mishawaka, IN.

\section{References}

1. Elhakim TS, Abdul HS, Romero CP, Rodriguez-Fuentes Y. Spontaneous pneumomediastinum, pneumothorax and subcutaneous emphysema in COVID-19 pneumonia: a rare case and literature review. BMJ Case Rep. 2020;13(12):e239489.

2. Kouritas VK, Papagiannopoulos K, Lazaridis G, Baka S, Mpoukovinas I, Karavasilis V, Lampaki S, Kioumis I, Pitsiou G, Papaiwannou A, Karavergou A. Pneumomediastinum. J Thorac Dis. 2015;7(Suppl 1):S44.

3. Dajer-Fadel WL, Argüero-Sánchez R, Ibarra-Perez C, Navarro-Reynoso FP. Systematic review of spontaneous pneumomediastinum: a survey of 22 years' data. Asian Cardiovasc Thorac Ann. 2014;22(8):997-1002.

4. Murayama S, Gibo S. Spontaneous pneumomediastinum and Macklin effect: overview and appearance on computed tomography. World J Radiol. 2014;6(11):850.

5. Fleming AM, Zambetti BR, Valaulikar GS. Bedside Mediastinotomy for Tension Pneumomediastinum with Tamponade in COVID-19. Ann Thorac Surg. 2021.

Figures Legends

Figure 1. Computed axial tomography image in axial view of the lungs on admission demonstrated diffuse bilateral ground-glass airspace disease consistent with COVID-19 pneumonia.

Figure 2. Computed axial tomography images of the lungs upon readmission in (A) axial view, (B) coronal view, and (C) sagittal view demonstrating resolution of diffuse bilateral infiltrates from prior hospitalization. Also depicted is massive pneumomediastinum and subcutaneous air throughout the entirety of the visualized body. There is also a small right-sided pneumothorax.

Figure 3. Post-operative photo depicting anterior mediastinal and pericardial window Blake (24 French) drains exiting through the subxiphoid region. 

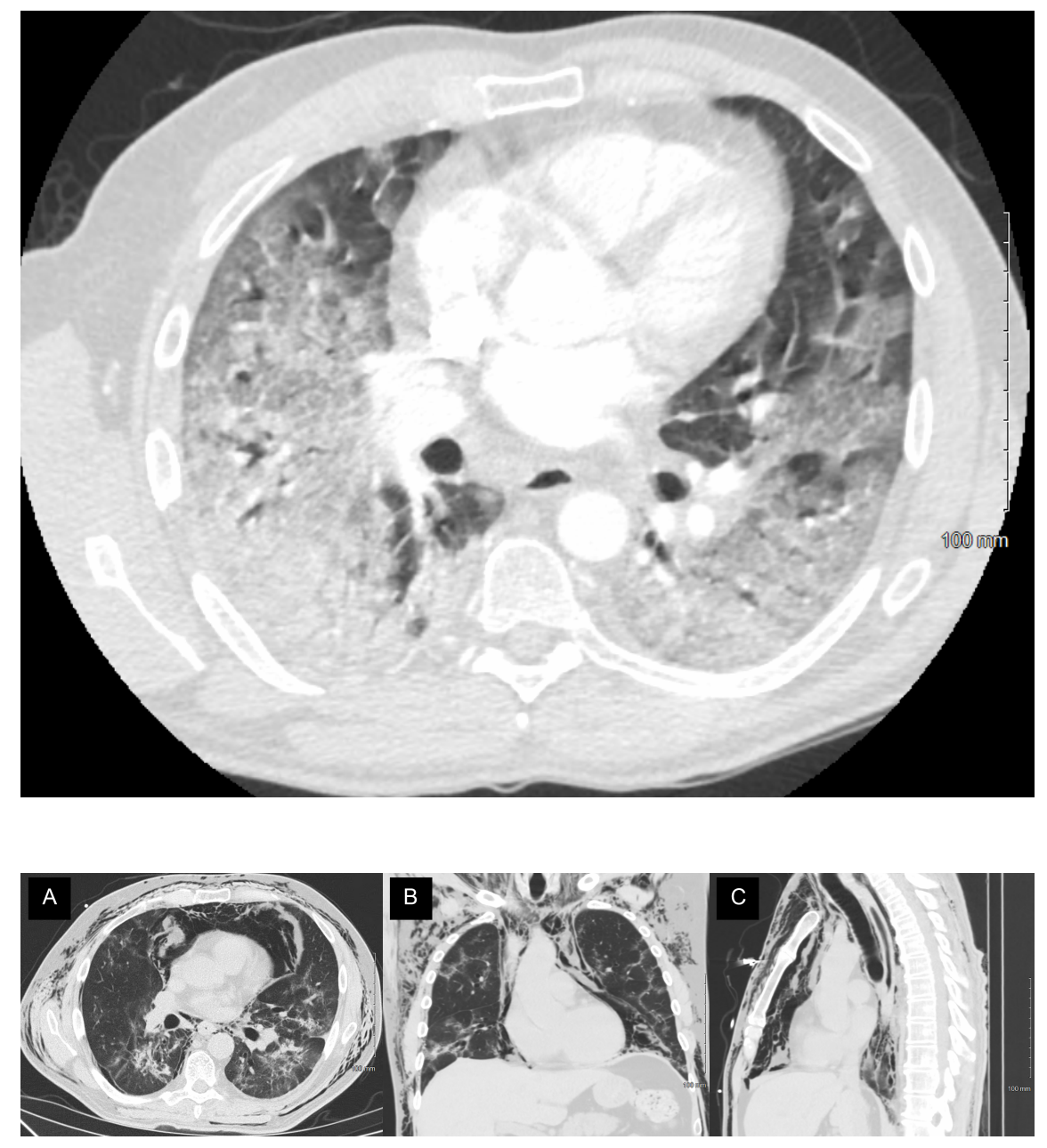


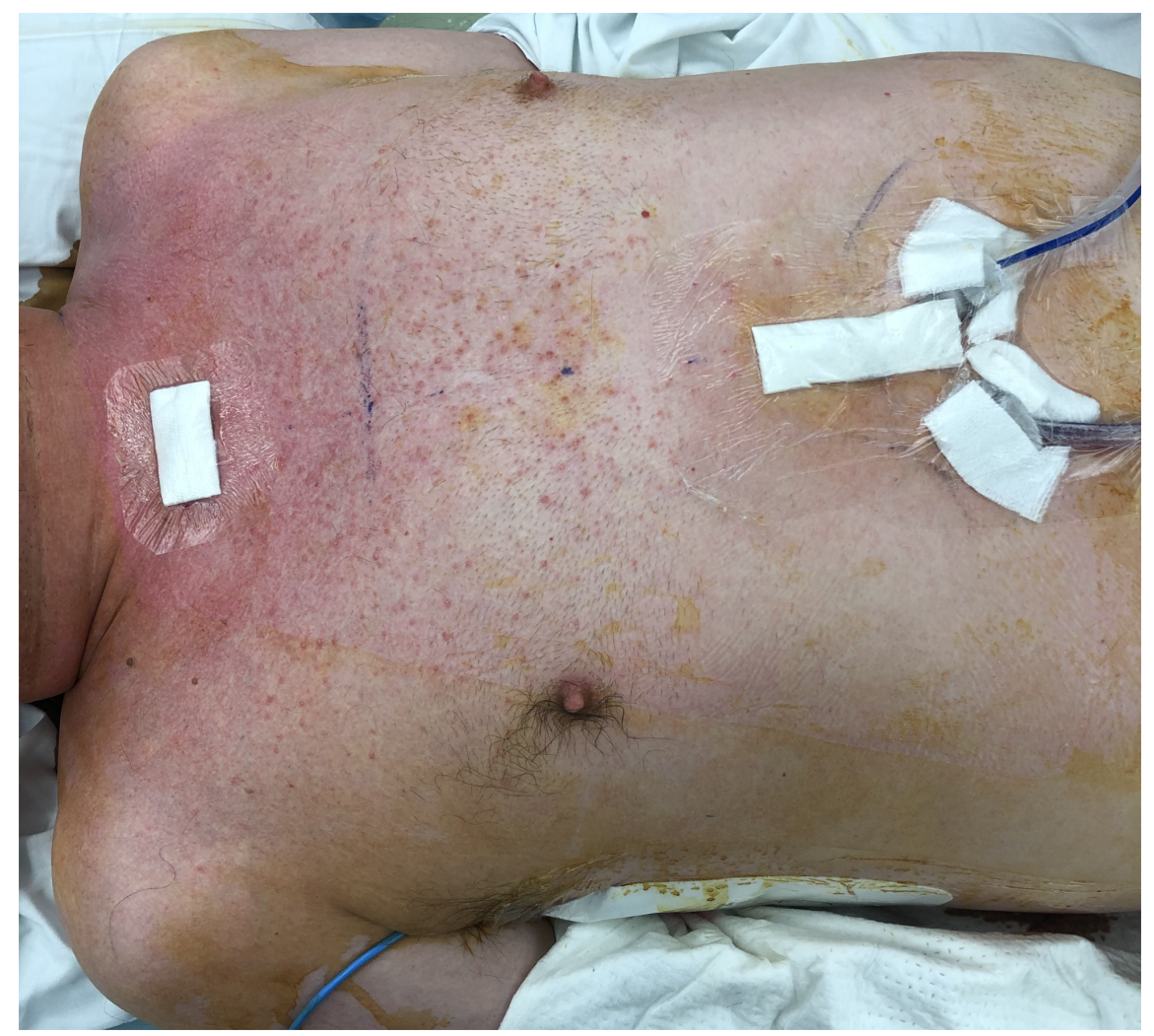

